\title{
Results of Numerical Modeling of the Origin of Cyclones and Anticyclones in the Vicinity of the Intertropical Convergence Zone
}

\author{
Igor V. Mingalev, Konstantin G. Orlov, Victor S. Mingalev* \\ Polar Geophysical Institute, Russian Academy of Sciences, Apatity, Russia \\ Email: *mingalev@pgia.ru
}

How to cite this paper: Mingalev, I.V., Orlov, K.G. and Mingalev, V.S. (2019) Results of Numerical Modeling of the Origin of Cyclones and Anticyclones in the Vicinity of the Intertropical Convergence Zone. Atmospheric and Climate Sciences, 9, 213-228.

https://doi.org/10.4236/acs.2019.92015

Received: January 29, 2019

Accepted: March 17, 2019

Published: March 20, 2019

Copyright $\odot 2019$ by author(s) and Scientific Research Publishing Inc. This work is licensed under the Creative Commons Attribution International License (CC BY 4.0).

http://creativecommons.org/licenses/by/4.0/

\begin{abstract}
A review of simulation results, devoted to time-dependent modeling of the initial stage of the formation of large-scale vortices in the troposphere in the vicinity of the intertropical convergence zone, is presented. The simulation results were obtained not long ago with the help of the mathematical model of the neutral wind system of the lower atmosphere, developed earlier in the Polar Geophysical Institute. The utilized mathematical model produces three-dimensional distributions of the atmospheric parameters in the height range from 0 to $15 \mathrm{~km}$ over a limited region of the Earth's surface. Simulation results were obtained for the case when the limited three-dimensional simulation domain, situated at low latitudes, is intersected by an intertropical convergence zone in the west-east direction. The reviewed simulation results were obtained for various initial configurations of the intertropical convergence zone. Results of numerical modeling have indicated that the origin of convexities in the form of the intertropical convergence zone can lead to the formation of different large-scale vortices in the lower atmosphere, in particular, a tropical cyclone, pair of cyclonic vortices, pair of cyclonic-anticyclonic vortexes, and triplet of cyclonic vortices. The simulation results, obtained earlier and presented individually in various editions, are reviewed and summarized in the present paper. A physical mechanism, responsible for the formation of the simulated large-scale vortices in the vicinity of the intertropical convergence zone, is discussed.
\end{abstract}

\section{Keywords}

Numerical Simulation, Air Flow, Lower Atmosphere, Tropical Cyclones

\section{Introduction}

An investigation of physical mechanisms responsible for the initial formation of 
tropical cyclones is a very important problem. We know that severe tropical cyclonic storms and hurricanes can cause tremendous damage and numerous fatalities. Consequently, prediction of tropical cyclone formation is a very important task. However, a physical theory of tropical cyclone origin is still far from completion. Unfortunately, to understand the physical mechanisms responsible for the initial formation of tropical cyclones, detailed three-dimensional fields of thermodynamical and gas dynamical parameters of the lower atmosphere can't be measured with sufficient accuracy with the help of modern scientific facility. Luckily, to investigate the physical mechanisms responsible for the initial formation of tropical cyclones, not only the theoretical and experimental but also computational studies may be applied. In particular, a tropical cyclone formation from pre-existing large-scale disturbances of the troposphere, or from vortices which are precursors to tropical cyclones, was numerically investigated earlier (for example, see [1]-[8]). Nevertheless, many of the details of the initial stage of the origin of tropical cyclones are still unresolved.

In the Polar Geophysical Institute (PGI), the mathematical model of the neutral wind system of the lower atmosphere, has been developed not long ago [9]. This mathematical model was utilized for obtaining the simulation results to be shown in the presented paper below. It can be noticed that the utilized mathematical model is the regional version of the non-hydrostatic mathematical model of the global wind system in the Earth's atmosphere, developed earlier in the PGI [10] [11] and applied for numerical investigations of the formation of the atmospheric global circulation under different geophysical conditions [12] [13] [14] [15] [16]. The mathematical model of the neutral wind system of the lower atmosphere, utilized in the presented study, was applied for time-dependent modeling of the initial stage of the formation of polar lows in the vicinity of the arctic front [17] [18], also.

In essence, the mathematical model, utilized in the presented study, was applied for numerical investigations of the initial stage of the formation of tropical cyclones [19]-[25]. These investigations have indicated that the origin of convexities in the form of the intertropical convergence zone, having the specific configurations, can lead to the formation of different large-scale vortices in the vicinity of the intertropical convergence zone, during the period not longer than three days. The fact is that the results of the investigations, devoted to time-dependent modeling of the initial stage of the formation of cyclones, were presented in various editions and reported at different conferences, individually. The purpose of the present study is to summarize and discuss all these results in one paper.

\section{Brief Description of the Utilized Mathematical Model}

In the utilized mathematical model, the atmospheric gas is considered as a mixture of air and water vapor, in which two types of precipitating water (namely, water microdrops and ice microparticles) can exist. The model is based on the numerical solution of the system of transport equations containing the equations 
of continuity for air and for the total water content in all phase states, momentum equations for the zonal, meridional, and vertical components of the air velocity, and energy equation. The characteristic feature of the model is that the vertical component of the air velocity is calculated without using the hydrostatic equation. Instead, the vertical component of the air velocity is obtained by means of a numerical solution of the appropriate momentum equation, with whatever simplifications of this equation being absent. In the momentum equations for all components of the air velocity, the effect of the turbulence on the mean flow is taken into account by using an empirical subgrid-scale parameterization similarly to the global circulation model of the Earth's atmosphere developed earlier in the PGI [10] [11].

Thus, the utilized mathematical model is based on numerical solving of non-simplified gas dynamic equations and produces three-dimensional timedependent distributions of the wind components, temperature, air density, water vapor density, concentration of micro drops of water, and concentration of ice particles. The model takes into account heating/cooling of the air due to absorption/emission of infrared radiation, as well as due to phase transitions of water vapor to micro drops of water and ice particles, which play an important role in energetic balance.

The three-dimensional simulation domain of the model is a part of a spherical layer stretching from land and ocean surface up to the altitude of $15 \mathrm{~km}$ over a limited region of the Earth's surface. In the initial applications of the model, the dimensions of the simulation domain in longitudinal and latitudinal directions were $32^{\circ}$ and $25^{\circ}$, respectively. The finite-difference method and explicit scheme are applied for solving the system of governing equations. The calculated parameters are determined on a uniform grid. The latitude and longitude steps are equal to $0.08^{\circ}$, and height step is equal to $200 \mathrm{~m}$. Complete details of the utilized finite-difference method and numerical schemes have been presented in the paper of Mingalev et al. [26]. More complete details of the utilized mathematical model may be found in the studies of Belotserkovskii et al. [9] [19] and Mingalev et al. [23].

\section{Presentation and Discussion of Results}

To investigate the initial stage of the formation of large-scale vortices in the vicinity of an intertropical convergence zone, we located the three-dimensional simulation domain at tropical latitudes. Simulation results were obtained for the cases when the simulation domain was intersected by an intertropical convergence zone in the west-east direction. It is known that an intertropical convergence zone may be considered as a fluid stream, having enhanced zonal velocities, in the ambient atmospheric gas, with a zonal flow of air being westward. A meridional wind velocity directs towards the centerline of an intertropical convergence zone at levels less than approximately $3 \mathrm{~km}$ and directs from the centerline of an intertropical convergence zone at levels higher than approximately $3 \mathrm{~km}$. A vertical wind velocity in an intertropical convergence zone is upward. 
The width of an intertropical convergence zone can achieve a value of some hundreds of kilometers.

For studying the behavior of an intertropical convergence zone, satellite microwave observations may be applied, in particular, the data including global radio thermal fields of the Earth at the frequencies containing the information about a moisture and water integral content distribution in the troposphere. Such observations indicate that the form of an intertropical convergence zone may be different and, sometimes, can contain convexities with distinct shapes. The north-south position of the intertropical convergence zone responds to changes in interhemispheric temperature contrast. An asymmetry in air-sea interactions can play an important role in forming the configuration of the intertropical convergence zone [27] [28] [29].

The authors of the study by Mingalev et al. [23] have investigated many results of satellite microwave monitoring of the Earth's atmosphere, obtained in Space Research Institute of the Russian Academy of Sciences and included in the electronic collection "GLOBAL-Field" (http://www.iki.rssi.ru/asp). As a consequence of these investigations, the authors of the mentioned study have advanced a hypothesis of the important role of the shape of the intertropical convergence zone on the process of the formation of tropical cyclones. To verify the advanced hypothesis, model simulations were performed with the help of the mathematical model of the neutral wind system of the lower atmosphere, briefly described in the previous Section. Simulation results were obtained for various cases in which the initial forms of the intertropical convergence zone were different and contained convexities with distinct shapes. For these cases, the south boundary of the simulation domain has been disposed in the vicinity of the equator. It was supposed that the simulation domain was located between $5^{\circ} \mathrm{S}$ and $20^{\circ} \mathrm{N}$ and situated over the Atlantic ocean.

Let us consider the simulation results, obtained for the case when, at the initial moment, the intertropical convergence zone contains a convexity in the north direction, with the deviation achieving a value of a few hundreds of kilometers [23]. The initial form of the intratropical convergence zone may be easy seen from the top panel of Figure 1, where it is like a light curved band. It is essential to note that, in the considered case, the west crook of the convexity is sharp while the east crook of the convexity is gently sloping, with the west and east ends of the convexity being at the same latitudes.

The time evolution of model parameters was numerically simulated using the mathematical model during the period for about one day. The results of time-dependent modeling are partly shown in Figure 1. As can be seen from this figure, in the course of time, the initial distribution of horizontal component of the air velocity was considerably transformed. A cyclonic vortex flow arose whose center is close to the southern edge of the initial intertropical convergence zone. The horizontal wind velocity in this cyclone achieved a value of $20 \mathrm{~m} / \mathrm{s}$ during the period of twenty seven hours. The radius of this large-scale cyclonic vortex is about $600 \mathrm{~km}$. 


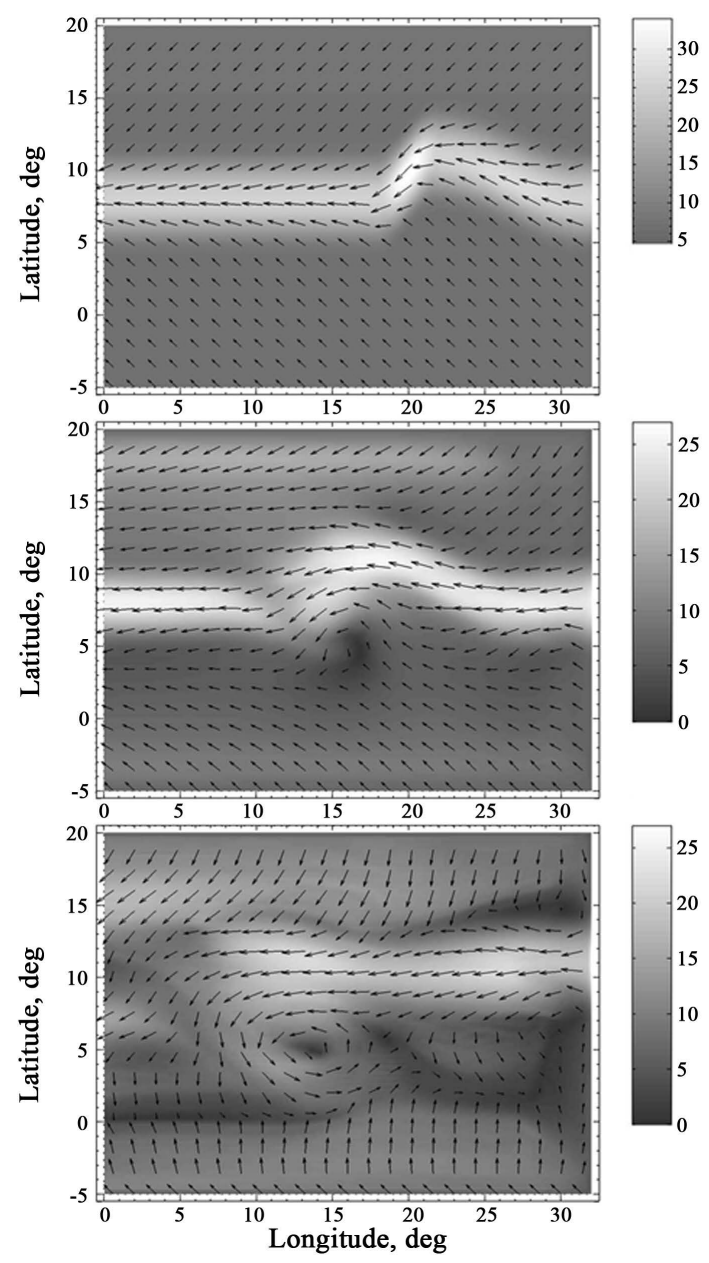

Figure 1. The distributions of horizontal component of the air velocity $(\mathrm{m} / \mathrm{s})$ at the altitude of $600 \mathrm{~m}$, assigned at the initial moment (top panel), computed 12 hours after the beginning of calculations (middle panel), and computed 27 hours after the beginning of calculations (lowest panel) [23]. The degree of shadowing of the figures indicates the module of the velocity in $\mathrm{m} / \mathrm{s}$.

In Figure 2, an exaggerated representation of the simulation results, presented in Figure 1, is shown which were assigned at the initial moment and computed 27 hours after the beginning of calculations. Figure 2 is intended for schematic representation of the simulation results at the altitude of $600 \mathrm{~m}$. Its top panel indicates the initial shape of the intertropical convergence zone. Its lower panel shows the cyclonic vortex arisen at the end of the simulation process (27 hours after the beginning of calculations).

From Figure 2, one has to see that the origin of a convexity in the configuration of the intratropical convergence zone can lead to the formation of a cyclone during the period for about one day. The center of this cyclone is close to the southern edge of the initial intertropical convergence zone.

A physical reason of the formation of the calculated tropical cyclone is the origin of a convexity in the configuration of the intertropical convergence zone. The origin of a convexity of the intertropical convergence zone leads to beginning 

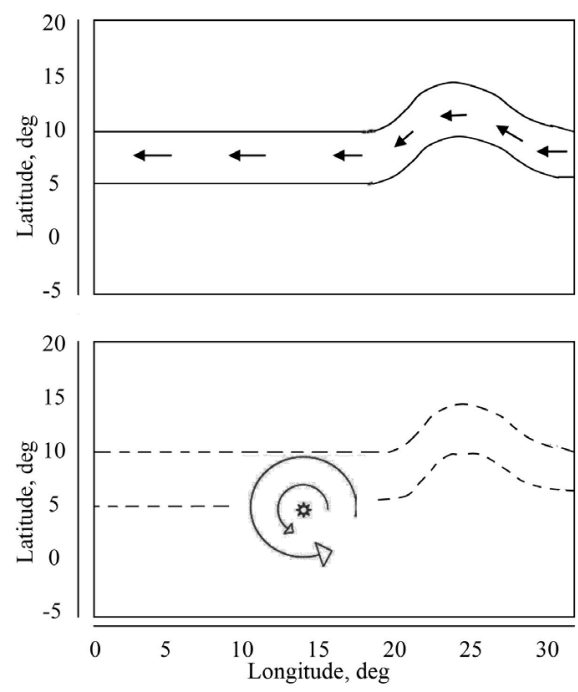

Figure 2. An exaggerated representation of the simulation results, presented in Figure 1 and taken from [23]. The initial configuration of the intertropical convergence zone (top panel) and the arisen cyclone position corresponding to 27 hours after the beginning of calculations (lower panel).

of an instability of stream air flow. As a consequence, a large-scale vortex flow arises in the lower atmosphere, with its center being close to the southern edge of the initial intertropical convergence zone. When the mixture of air and water vapor moves to higher altitudes, where temperature is lower, energy, emitted due to phase transitions of water vapor to micro drops of water and ice particles, transforms into kinetic energy of the air flow, with the horizontal wind velocity increasing in formed cyclonic vortex.

In the study of Mingalev et al. [23], the simulation results have been presented which were obtained for another case when the initial form of the intertropical convergence zone contained the convexity in the north direction, with the east end of the convexity being situated at more northern latitudes than the west end of the convexity (see top panel of Figure 3). The results of numerical modeling have indicated that, in this case, in the course of time, cyclonic vortex flow arose whose center is close to the southern edge of the initial intertropical convergence zone. This fact is schematically shown in the lower panel of Figure 3. It is easy to see that the simulation results, presented on lower panel of Figure 2, are similar to the results of numerical modeling, presented on lower panel of Figure 3.

Also, in the study of Mingalev et al. [23], the results of numerical modeling have been presented which were obtained for the case when the initial form of the intertropical convergence zone contained the convexity in the north direction, with the east end of the convexity being situated at more southern latitudes than the west end of the convexity (see top panel of Figure 4). In this case, the simulation results have indicated that, in the course of time, a cyclon arose whose center is close to the southern edge of the initial intertropical convergence zone. This fact is schematically shown in the lower panel of Figure 4. It can be noticed that, after 27 hours of calculations, the center of the cyclonic vortex, 

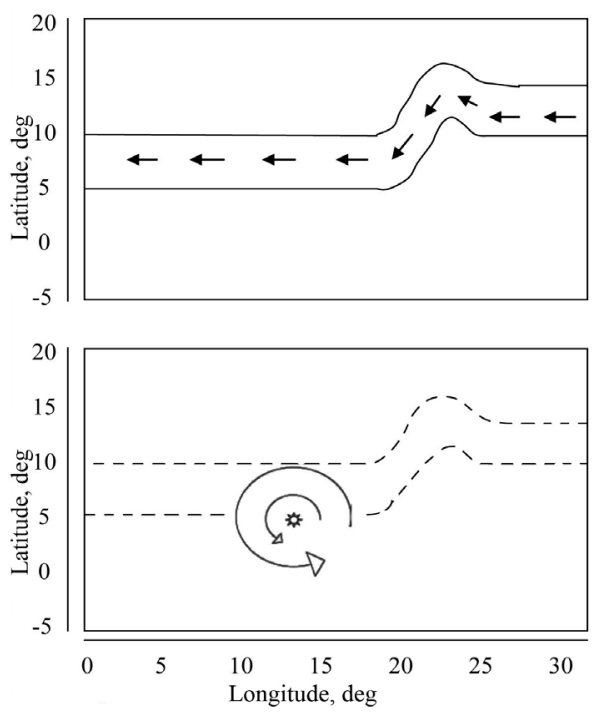

Figure 3. A schematic representation of the simulation results, taken from [23], at the altitude of $600 \mathrm{~m}$. The initial configuration of the intertropical convergence zone (top panel) and the arisen cyclone position corresponding to 27 hours after the beginning of calculations (lower panel).
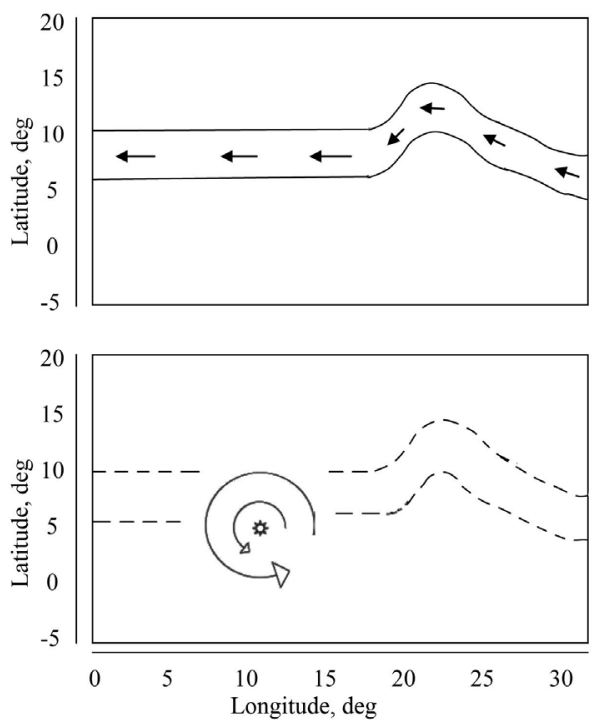

Figure 4. A schematic representation of the simulation results, taken from [23], at the altitude of $600 \mathrm{~m}$. The initial configuration of the intertropical convergence zone (top panel) and the arisen cyclone position corresponding to 27 hours after the beginning of calculations (lower panel).

arisen in the considered case, is situated at more western longitude than the centers of the cyclones, arisen in the previous two cases.

It may be recalled that, in three cases considered above, the initial forms of the intertropical convergence zone were different and contained convexities with distinct shapes. However, these shapes have a common feature, namely, the west crooks of the convexities are sharp while the east crooks of the convexities are gently sloping. 
Let us consider the fourth case when, at the initial moment, west and east crooks of the convexity are sharp and located at the same latitudes, with the deviation of the convexity being in the north direction [24]. The initial form of the intertropical convergence zone may be easy seen from the top panel of Figure 5. In this case, the simulation results have indicated that, in the course of time, a pair of cyclonic-anticyclonic vortexes arose (see the lower panel of Figure 5). The center of the arisen cyclone is situated inside the initial intertropical convergence zone while the center of the arisen anticyclone is situated at more northern latitudes. Moreover, the arisen cyclone is situated at more western longitudes than the arisen anticyclone.

In the study of Mingalev et al. [24], the results of numerical simulation have been presented which were obtained for the case when, at the initial moment, the intertropical convergence zone contains a convexity, analogous to the convexity of the fourth case, but deviated in the south direction. In the top panel of Figure 6, the initial form of the intertropical convergence zone may be seen for considered fifth case. In this case, the results of numerical simulation have indicated that, in the course of time, a cyclone and anticyclone arose in the vicinity of the intertropical convergence zone (see the lower panel of Figure 6). The center of the arisen anticyclone is situated inside the initial intertropical convergence zone while the center of the arisen cyclone is situated at more southern latitudes. Moreover, the arisen cyclone is situated at more eastern longitudes than the arisen anticyclone.

It can be noticed that, in the large-scale vortexes, arisen in the fourth and fifth cases, the horizontal wind velocity achieved a value of $20 \mathrm{~m} / \mathrm{s}$ during the period of twenty seven hours. The radii of these large-scale vortexes are about $400 \mathrm{~km}$.
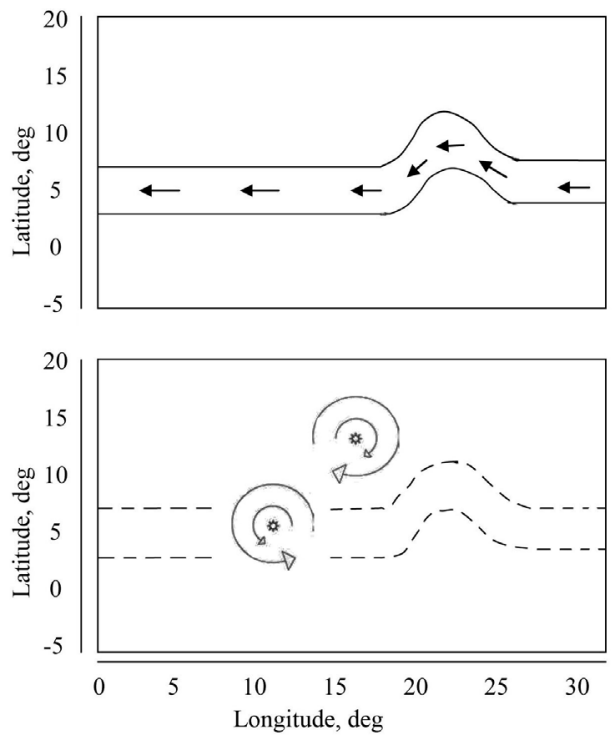

Figure 5. A schematic representation of the simulation results, taken from [24], at the altitude of $600 \mathrm{~m}$. The initial configuration of the intertropical convergence zone (top panel) and the position of a pair of cyclonic-anticyclonic vortexes at the moment corresponding to 27 hours after the beginning of calculations (lower panel). 

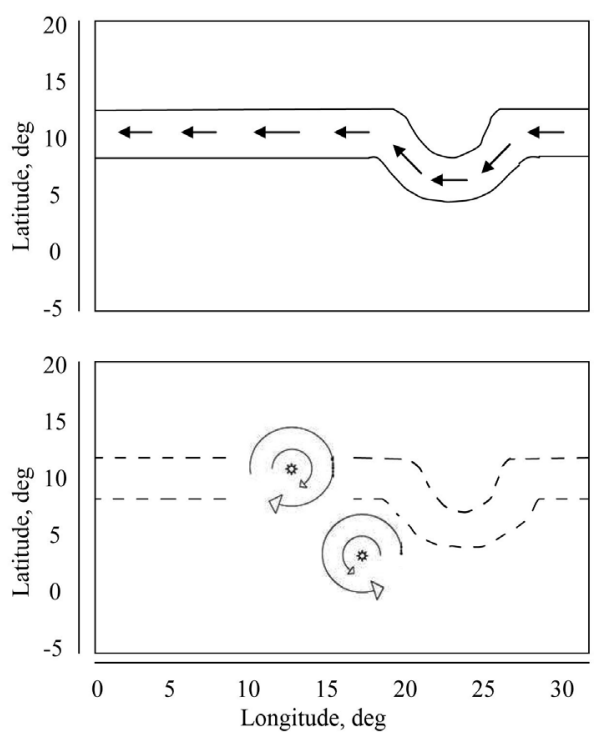

Figure 6. A schematic representation of the simulation results, taken from [24]. The initial configuration of the intertropical convergence zone (top panel) and the positions of the arisen cyclone and anticyclone at the moment corresponding to 27 hours after the beginning of calculations (lower panel).

Let us consider the sixth case when, at the initial moment, the intertropical convergence zone contains a convexity in the north direction, analogous to the convexity of the first case. However, in the first case, at the initial moment, the horizontal velocity field was approximately symmetric relatively the centerline of the intertropical convergence zone not only inside it but also beyond the intertropical convergence zone. Unlike, in the considered sixth case, at the initial moment, the horizontal velocity field is not symmetric relatively the centerline of the intertropical convergence zone inside and beyond it. Namely, the zonal wind velocities at more northern latitudes relatively the centerline of the intertropical convergence zone are larger than at more southern latitudes relatively it.

The time evolution of model parameters was numerically simulated using the mathematical model during the period for about three days [24]. The results of time-dependent modeling are schematically shown in Figure 7. The results of numerical simulation have indicated that, in the course of time, a pair of cyclonic vortices arose in the vicinity of the intertropical convergence zone. The rotational centers of these cyclonic vortices are situated inside the initial intertropical convergence zone. The horizontal wind velocity in these cyclonic vortices achieved values of $15-20 \mathrm{~m} / \mathrm{s}$ during the period of seventy hours. The radii of these cyclonic vortices are in the $500-600 \mathrm{~km}$ range.

It should be emphasized that the results of satellite monitoring of the Earth's atmosphere often indicated an origin of not only a single cyclone or a single anticyclone but also a simultaneous origin of a cyclone-anticyclone pair as well as a simultaneous origin of a pair of cyclonic vortices [30].

Now, let us consider the seventh case in which a modified version of the mathematical model was applied. In the modified version of the model, the dimension 

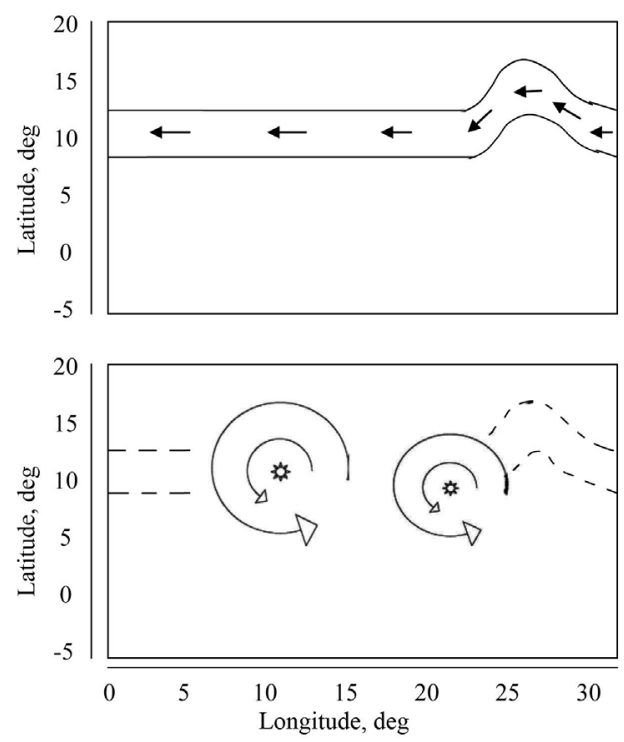

Figure 7. A schematic representation of the simulation results, taken from [24]. The initial configuration of the intertropical convergence zone (top panel) and the position of the arisen pair of cyclonic vortices at the moment corresponding to 70 hours after the beginning of calculations (lower panel).

of the simulation domain in longitudinal direction has been enlarged to $44^{\circ}$ [22]. In the considered case, at the initial moment, the eastern part of the intertropical convergence zone contains a convexity in the north direction, analogous to the convexity of the first case. However, in the first case, at the initial moment, the western part of the intertropical convergence zone was parallel to the equator. Unlike, in the considered seventh case, at the initial moment, the western part of the intertropical convergence zone is displaced from the equator to the north when the longitude is increased (see the top panel of Figure 8).

Time-dependent modeling was performed, using the mathematical model, during the period of about 60 hours. The results of numerical simulation indicated that, in the course of time, a triplet of cyclonic vortices arose in the vicinity of the intertropical convergence zone. After the origin, all cyclonic vortices have moved in the western direction. The results of time-dependent modeling are schematically shown in the lower panel of Figure 8 at the moment corresponding to 60 hours after the beginning of calculations. The rotational centers of two cyclonic vortices are situated inside the initial intertropical convergence zone. The rotational center of third cyclone is situated close to the southern edge of the initial intertropical convergence zone. The horizontal wind velocity in these cyclonic vortices achieved values of $15-18 \mathrm{~m} / \mathrm{s}$ during the period of sixty hours. The radii of these cyclonic vortices are in the $450-600 \mathrm{~km}$ range.

In the study of Mingalev et al. [25], the results of numerical simulation have been presented which were obtained with the help of a modified version of the mathematical model in which the dimension of the simulation domain in longitudinal direction has been enlarged to $75^{\circ}$. The results of numerical simulation have been obtained for the case when, at the initial moment, the intertropical 
convergence zone contains two convexities in the north direction, with the deviations achieving a value of a few hundreds of kilometers. The initial form of the intertropical convergence zone for considered eighth case may be seen in the top panel of Figure 9.

The time evolution of model parameters was numerically simulated using the mathematical model during the period of about four days. The results of simulation indicated that, in the course of time, the initial distribution of horizontal component of the air velocity was considerably transformed. In a moment of 20 hours after the beginning of calculations, a pair of tropical cyclonic vortices arose. Their centers were close to the southern edge of the initial intertropical convergence zone. In a moment of 50 hours after the beginning of calculations, these cyclonic vortices have moved in the western direction for about 10 degrees.

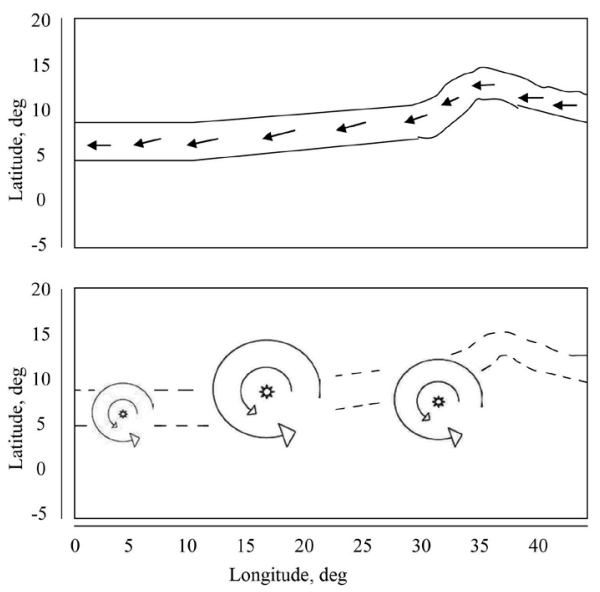

Figure 8. A schematic representation of the simulation results, taken from [22]. The initial configuration of the intertropical convergence zone (top panel) and the position of the arisen triplet of cyclonic vortices at the moment corresponding to 60 hours after the beginning of calculations (lower panel).

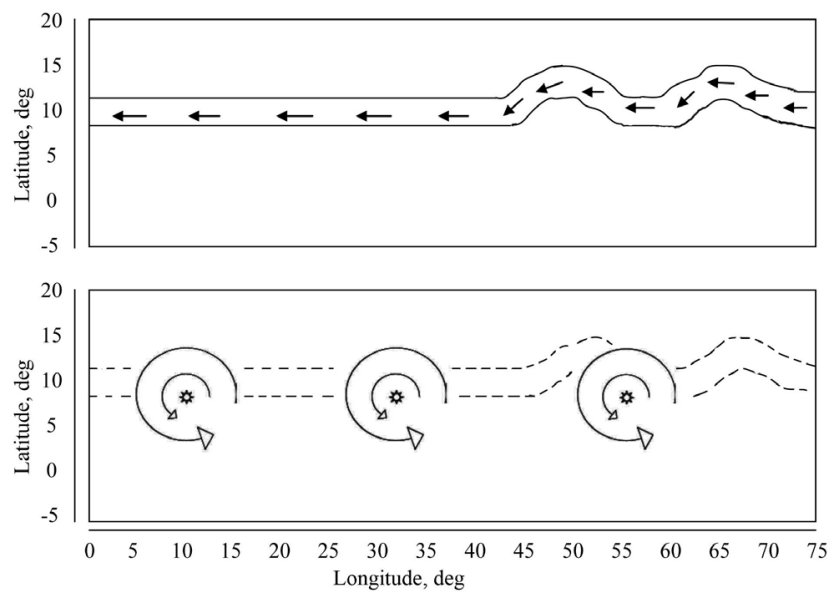

Figure 9. A schematic representation of the simulation results, taken from [25]. The initial configuration of the intertropical convergence zone (top panel) and the position of the arisen triplet of cyclonic vortices at the moment corresponding to 90 hours after the beginning of calculations (lower panel). 
Simultaneously, the third cyclonic vortex arose, with its center having been close to the southern edge of the initial intertropical convergence zone. All arisen cyclonic vortices have moved in the western direction. The displacement of third cyclonic vortex is about 6 degrees at a moment of 90 hours after the beginning of calculations. For this moment, the results of simulation are schematically shown in the lower panel of Figure 9. The horizontal wind velocity in the cyclones can achieve values of $15-20 \mathrm{~m} / \mathrm{s}$ in the course of time. The maximum wind velocities within the vortices were reached approximately $20 \mathrm{~h}$ after their origins, and then they began to decrease slowly. The radii of these three cyclones were about $800 \mathrm{~km}$.

\section{Summary and Concluding Remarks}

Earlier, a series of articles, devoted to an investigation of the initial stage of the formation of large-scale vortices in the vicinity of the intertropical convergence zone, have been published in various editions. The results, presented in these articles, were obtained with the help of a numerical simulation. In these articles, it was shown that a change of the configuration of the intertropical convergence zone can influence the process of the formation of tropical cyclones. Authors of the present paper made an attempt to review and summarize the results, included in the previous articles and obtained by numerical modeling.

The reviewed results were obtained with the help of a mathematical model of the neutral wind system of the lower atmosphere, developed in the Polar Geophysical Institute. The utilized mathematical model produces time-dependent three-dimensional distributions of the atmospheric parameters in the height range from 0 to $15 \mathrm{~km}$ over a limited region of the Earth's surface. Simulation results were obtained for the cases when the limited three-dimensional simulation domain is intersected by an intertropical convergence zone in the west-east direction. The reviewed simulation results were obtained for various initial configurations of the intertropical convergence zone. Results of time-dependent numerical modeling have indicated that the origin of convexities in the form of the intertropical convergence zone can lead to the formation of different large-scale vortices in the lower atmosphere.

Firstly, the simulation results were reviewed which were obtained for the cases when the initial form of the intertropical convergence zone contained the convexities in the north direction, with the west crooks of the convexities being sharp while the east crooks of the convexities being gently sloping. In these cases, in the course of time, a single cyclonic vortex arose whose center is close to the southern edge of the initial intertropical convergence zone.

Secondly, the results of simulation were considered for the cases when, at the initial moment, west and east crooks of the convexity were sharp, with the deviations of the convexities being both in the north direction and in the south direction. In these cases, the simulation results have indicated that, in the course of time, a pair of cyclonic-anticyclonic vortexes arose, with the center of the arisen 
anticyclone being situated at more northern latitudes than the center of the arisen cyclone.

Thirdly, the simulation results were discussed for the case when, at the initial moment, the intertropical convergence zone contains a convexity in the north direction, however, the horizontal velocity field was not symmetric relatively the centerline of the intertropical convergence zone inside and beyond it. In this case, the simulation results have indicated that, in the course of time, a pair of cyclonic vortices arose in the vicinity of the intertropical convergence zone, with the rotational centers of these cyclonic vortices being situated inside the initial intertropical convergence zone.

Fourthly, the results of numerical simulation have been presented which were obtained with the help of a modified version of the mathematical model in which the dimension of the simulation domain in longitudinal direction has been enlarged. It turned out that there are initial configurations of the intertropical convergence zone, which can lead to the formation of a triplet of cyclonic vortices in the vicinity of the initial intertropical convergence zone in the course of time.

It should be emphasized that the results of satellite monitoring of the Earth's atmosphere often indicate that the form of an intertropical convergence zone may be different and, sometimes, can contain convexities with distinct shapes. These convexities can play a rule of pre-existing large-scale disturbances of the troposphere for an origin of cyclones and anticyclones in the vicinity of the intertropical convergence zone.

A physical reason of the formation of the calculated tropical large-scale vortices in the vicinity of the intertropical convergence zone is a rise of instability of stream air flow present in this zone. The cause for the rise of this instability is the origin of the convexities in the configuration of the intertropical convergence zone. This instability leads to considerable transformation of the air flow. As a result, the intertropical convergence zone may be broken and tropical large-scale vortices can be formed in the vicinity of the initial position of the intertropical convergence zone in the course of time. Asymmetry of the horizontal velocity fields relatively the centerline of the intertropical convergence zone inside and beyond it can influence the process of the formation of different large-scale vortices in the lower atmosphere. The transformation of the energy, emitted due to phase transitions of water vapor to micro drops of water and ice particles, into kinetic energy of the air flow can lead to an acceleration of this flow.

Incidentally, according to observations, not each cyclonic or anticyclonic vortex, arisen in the lower atmosphere, has the potential to grow up to the long-live large-scale atmospheric vortex. It is known that, sometimes, a vortex, initially arisen in the lower atmosphere, can be attenuated in the course of time and will not achieve a status of the long-live large-scale atmospheric vortex.

Nevertheless, it can be noticed that simulation results, reviewed in the present study, may be applied to constructing an effective method of early prediction of tropical cyclones and hurricanes origination, based on the analysis of satellite 
observational data, dealing with the configuration of the intertropical convergence zone.

\section{Acknowledgements}

This work was partly supported by Grant No. 18-29-03022 from the Russian Foundation for Basic Research.

\section{Conflicts of Interest}

The authors declare no conflicts of interest regarding the publication of this paper.

\section{References}

[1] Ooyama, K. (1969) Numerical Simulation of the Life Cycle of Tropical Cyclones. Journal of Atmospheric Sciences, 26, 3-40. https://doi.org/10.1175/1520-0469(1969)026<0003:NSOTLC $>2.0 . C O ; 2$

[2] Montgomery, M.T. and Enagonio, J. (1998) Tropical Cyclogenesis via Convectively Forced Vortex Rossby Waves in a Three-Dimensional Quasigeostrophic Model. Journal of Atmospheric Sciences, 55, 3176-3207. https://doi.org/10.1175/1520-0469(1998)055<3176:TCVCFV>2.0.CO;2

[3] Li, T., Ge, X., Wang, B. and Zhu, Y. (2006) Tropical Cyclogenesis Associated with Rossby Wave Energy Dispersionof a Preesxisting Typhoon. Part II: Numerical Simulations. Journal of Atmospheric Sciences, 63, 1390-1409.

https://doi.org/10.1175/JAS3693.1

[4] Montgomery, M.T., Wang, Z. and Dunkerton, T.J. (2010) Coarse, Intermediate and High Resolution Numerical Simulation of the Transition of a Tropical Wave Critical Layer to a Tropical Storm. Atmospheric Chemistry and Physics, 10, 10803-10827. https://doi.org/10.5194/acp-10-10803-2010

[5] Venkatesh, T.N. and Mathew, J. (2010) A Numerical Study of the Role of the Vertical Structure of Vorticity during Tropical Cyclone Genesis. Fluid Dynamics Research, 42, Article ID: 045506. https://doi.org/10.1088/0169-5983/42/4/045506

[6] Reed, K.A. and Jablonowski, C. (2011) Impact of Physical Parameterizations on Idealized Tropical Cyclones in the Community Atmosphere Model. Geophysical Research Letters, 38, Article ID: L04805. https://doi.org/10.1029/2010GL046297

[7] Abarca, S.F. and Corbosiero, K.L. (2011) Secondary Eyewall Formation in WRF Simulations of Hurricanes Rita and Katrina (2005). Geophysical Research Letters, 38, Article ID: L07802. https://doi.org/10.1029/2011GL047015

[8] Xu, Y.M. (2011) The Genesis of Tropical Cyclone Bilis (2000) Associated with Cross-Equatorial Surges. Advances in Atmospheric Sciences, 28, 665-681. https://doi.org/10.1007/s00376-010-9142-Z

[9] Belotserkovskii, O.M., Mingalev, I.V., Mingalev, V.S., Mingalev, O.V. and Oparin, A.M. (2006) Mechanism of the Appearance of a Large-Scale Vortex in the Troposphere above a Nonuniformly Heated Surface. Doklady Earth Sciences, 411, 1284-1288. https://doi.org/10.1134/S1028334X06080277

[10] Mingalev, I.V. and Mingalev, V.S. (2005) The Global Circulation Model of the Lower and Middle Atmosphere of the Earth with a Given Temperature Distribution. Mathematical Modeling, 17, 24-40. (In Russian)

[11] Mingalev, I.V., Mingalev, V.S. and Mingaleva, G.I. (2007) Numerical Simulation of 
Global Distributions of the Horizontal and Vertical Wind in the Middle Atmosphere Using a Given Neutral Gas Temperature Field. Journal of Atmospheric and Solar-Terrestrial Physics, 69, 552-568. https://doi.org/10.1016/j.jastp.2006.10.005

[12] Mingalev, I.V., Mingalev, O.V. and Mingalev, V.S. (2008) Model Simulation of Global Circulation in the Middle Atmosphere for January Conditions. Advances in Geosciences, 15, 11-16. https://doi.org/10.5194/adgeo-15-11-2008

[13] Mingalev, I.V., Mingalev, V.S. and Mingaleva, G.I. (2012) Numerical Simulation of the Global Neutral Wind System of the Earth's Middle Atmosphere for Different Seasons. Atmosphere, 3, 213-228. https://doi.org/10.3390/atmos3010213

[14] Mingalev, I.V. and Mingalev, V.S. (2012) Numerical Modeling of the Influence of Solar Activity on the Global Circulation in the Earth's Mesosphere and Lower Thermosphere. International Journal of Geophysics, 2012, Article ID: 106035.

[15] Mingalev, I., Mingaleva, G. and Mingalev, V. (2013) A Simulation Study of the Effect of Geomagnetic Activity on the Global Circulation in the Earth's Middle Atmosphere. Atmospheric and Climate Sciences, 3, 8-19.

https://doi.org/10.4236/acs.2013.33A002

[16] Mingalev, I.V., Orlov, K.G. and Mingalev, V.S. (2017) Numerical Modeling of the Influence of the Relief of a Planet on the Global Circulation of the Earth's Stratosphere and Mesosphere. Atmospheric and Climate Sciences, 7, 496-510. https://doi.org/10.4236/acs.2017.74036

[17] Mingalev, I.V., Orlov, K.G. and Mingalev, V.S. (2012) A Mechanism of Formation of Polar Cyclones and Possibility of Their Prediction Using Satellite Observations. Cosmic Research, 50, 160-169. https://doi.org/10.1134/S0010952512010066

[18] Mingalev, I.V., Orlov, K.G. and Mingalev, V.S. (2014) A Modeling Study of the Initial Formation of Polar Lows in the Vicinity of the Arctic Front. Advances in Meteorology, 2014, Article ID: 970547.

[19] Belotserkovskii, O.M., Mingalev, I.V., Mingalev, V.S., Mingalev, O.V., Oparin, A.M. and Chechetkin, V.M. (2009) Formation of Large-Scale Vortices in Shear Flow of the Lower Atmosphere of the Earth in the Region of Tropical Latitudes. Cosmic Research, 47, 466-479. https://doi.org/10.1134/S0010952509060033

[20] Mingalev, I.V., Astafieva, N.M., Orlov, K.G., Mingalev, V.S. and Mingalev, O.V. (2010) The Mechanism of Formation of Cyclonic Vortices in the Intertropical Zone of Convergence and Their Early Detection. Current Problems in Remote Sensing of the Earth from Space, 7, 112-125. (In Russian)

[21] Mingalev, I.V., Astafieva, N.M., Orlov, K.G., Mingalev, V.S., Mingalev, O.V. and Chechetkin, V.M. (2011) Possibility of a Detection of Tropical Cyclones and Hurricanes Formation According to Satellite Remote Sensing. Current Problems in Remote Sensing of the Earth from Space, 8, 290-296. (In Russian)

[22] Mingalev, I.V., Astafieva, N.M., Orlov, K.G., Chechetkin, V.M., Mingalev, V.S. and Mingalev, O.V. (2012) Influence of a Geometry of Air Mass Flow in the Region of an Intertropical Zone of Convergence on Process of Formation of Cyclone Vortices. Current Problems in Remote Sensing of the Earth from Space, 9, 154-161. (In Russian)

[23] Mingalev, I.V., Astafieva, N.M., Orlov, K.G., Chechetkin, V.M., Mingalev, V.S. and Mingalev, O.V. (2012) Numerical Simulation of Formation of Cyclone Vortex Flows in the Intertropical Zone of Convergence and Their Early Detection. Cosmic Research, 50, 233-248. https://doi.org/10.1134/S0010952512020062

[24] Mingalev, I.V., Astafieva, N.M., Orlov, K.G., Mingalev, V.S., Mingalev, O.V. and Chechetkin, V.M. (2013) A Simulation Study of the Formation of Large-Scale Cyc- 
lonic and Anticyclonic Vortices in the Vicinity of the Intertropical Convergence Zone. ISRN Geophysics, 2013, Article ID: 215362.

[25] Mingalev, I.V., Astafieva, N.M., Orlov, K.G., Mingalev, V.S., Mingalev, O.V. and Chechetkin, V.M. (2014) Numerical Modeling of the Initial Formation of Cyclonic Vortices at Tropical Latitudes. Atmospheric and Climate Sciences, 4, 899-906. https://doi.org/10.4236/acs.2014.45079

[26] Mingalev, V.S., Mingalev, I.V., Mingalev, O.V., Oparin, A.M. and Orlov, K.G. (2010) Generalization of the Hybrid Monotone Second-Order Finite Difference Scheme for Gas Dynamics Equations to the Case of Unstructured 3D Grid. Computational Mathematics and Mathematical Physics, 50, 877-889. https://doi.org/10.1134/S0965542510050118

[27] Broccoli, A.J., Dahl, R.A. and Stouffer, R.J. (2006) Response of the ITCZ to Northern Hemisphere Cooling. Geophysical Research Letters, 33, L01702.

https://doi.org/10.1029/2005GL024546

[28] Fedorov, A., Barreiro, M., Boccaletti, G., Pacanowski, R. and Philander, S.G. (2007) The Freshening of Surface Waters in High Latitudes: Effects on the Thermohaline and Wind-Driven Circulations. Journal of Physical Oceanography, 37, 896-907. https://doi.org/10.1175/JPO3033.1

[29] Chiang, J.C.H. and Friedman, A.R. (2012) Extratropical Cooling, Interhemispheric Thermal Gradients, and Tropical Climate Change. Annual Review of Earth and Planetary Sciences, 40, 383-412. https://doi.org/10.1146/annurev-earth-042711-105545

[30] Chen, T.C., Tsay, J.D., Yen, M.C. and Cayanan, E.O. (2010) Formation of the Philippine Twin Tropical Cyclones during the 2008 Summer Monsoon Onset. Weather and Forecasting, 25, 1317-1341. https://doi.org/10.1175/2010WAF2222395.1 